\title{
Challenging Menstrual Norms in Online Medical Advice: Deconstructing Stigma through Entangled Art Practice
}

\author{
Bee Hughes 1,2*
}

Published: September 27, 2018

\begin{abstract}
This article analyses three examples of online medical advice provided by UK based health websites on the topic of menstruation, and reflects on my artistic practice as a critical response to notions of menstrual normativity. The article considers to what extent these online platforms - now part of the cultural fabric of contemporary healthcare advice - sustain dominant Western cultural perceptions of menstruation. Through thematic and comparative analysis, the article explores how these texts reflect cultural discourses around menstruation through reinforcing cis and heteronormative standards, presenting menstruation as failed pregnancy, and as a largely problematic rather than positive experience. The article also reflects upon autobiographical and performative artworks as spaces developed alongside the analysis of the online medical advice texts, which propose and explore resistance to the social stigma still associated with menstruating.
\end{abstract}

Keywords: autobiographical art, menstrual normativity, practice-led research, menstruation, gender

\section{INTRODUCTION}

This article considers three examples of online health advice available in 2018 aimed at a UK audience - NHS Choices, Patient.info and Boots WebMD - from a socio-cultural perspective. The analysis is presented within the context of my ongoing artistic practice, which is also discussed. The chosen example texts are from established reputable sources with direct links to sources of medical authority. While operational, Boots WebMD was a collaboration between the well-established USA health website WebMD and the UK pharmacy chain Boots, designed to provide basic information on health and wellbeing ${ }^{1}$. Patient.info is owned by Egton Medical Information Systems Ltd provides IT systems to UK General Practices. NHS Choices is published by the UK National Health Service and has UK Government Information Standard accreditation, and with 'more than 48 million visits per month' (NHS, 2015) has become the most visited UK health website (ibid). Patient.info differs from the other examples as it features two distinct strands of content, both authored and reviewed by medical professionals, but aimed at distinct user groups. For the purposes of this research, the analysis will be focussed on the 'Conditions' page, which is explicitly aimed at a lay audience as opposed to a clinical reader, in alignment with the other examples which are also produced with a general audience in mind. Artistic explorations have existed alongside and within menstrual activism since the 1970s (Bobel, 2007, 2010; Fahs, 2016; Johnston-Robledo and Chrisler, 2011) in addition to other forms of feminist art which have actively challenged the pervasive double-standards of sexism and misogyny (for example Jones, 1998, 2005; Rees, 2013). My research interest in menstruation stems from the development of an autobiographical artistic practice exploring my own experience of menstruating alongside theoretical enquiry into menstruation in everyday culture. Following the analysis of the example texts, I reflect upon my artistic practice as a method through which to deconstruct attitudes towards menstruation and to wilfully break the conventions of what Karen Houppert has called 'menstrual etiquette' (Houppert, 2000: 2). First, I explore notions of menstrual normativity through performative printmaking in my visual work, Cycles (2016-17), where I documented my menstrual cycle through body printing for six months. Second, I present an example of cut-up

${ }^{1}$ Boots WebMD remained live through the earlier research and drafting of this article. Since the initial submission, $W e b M D$ and Boots have ended their partnership, with little to no reporting or press information. The website can now be viewed via The Internet Archive, with the last 'live' snapshot saved to the archive's Way Back Macbine on May 10th 2018.

1 School of Humanities and Social Science, Liverpool John Moores University, UNITED KINGDOM

${ }^{2}$ Liverpool School of Art and Design, Liverpool John Moores University, UNITED KINGDOM

*Corresponding Author: B.Hughes@limu.ac.uk 
poetry, composed with — and as a challenge to — online texts such as those analysed in the first part of the article. Finally, I reflect upon translating poetic responses into collaborative soundworks, a process of re-embodying my emotional responses to the online medical advice explored. Following Rachel Allen's analysis of radical performance art in the context of critical medical humanities, the performative body explored in my visual work and fragmented text/voice of poetic experiments moves towards an image of the body 'hinged between nature and culture, 'the personal as political', objecthood and subjecthood, the biological and the psychical' (Allen, 2016: 195).

The selected online health resources are everyday medical texts, as they are specialised - dealing with specific areas of life, health and well-being - but are not specialist in that lay readers should be able to understand their content, they exist as part of everyday culture. The article explores how these texts reflect Western cultural experiences of menstruation, particularly considering how they might uphold deep-rooted cultural notions surrounding menstruation. Boots WebMD, NHS Choices and Patient.info, in their role as medical reference resources, are situated within a medical model of menstruation. However, as internet resources they sit outside the traditional boundaries of medical authority - for example the physical spaces of the consulting room or the intellectual space medical textbooks or research publications - and are a part of the fabric of our everyday interactions with the online world. The increasing use of online health advice (Smart and Burling, 2001) reflects the ubiquity of such technologies in every realm of everyday life and the impact of neoliberal ideology on therapeutic culture (Rose, 1999) and personal healthcare. As part of the cultural fabric of contemporary healthcare advice, it is pertinent to consider to what extent these platforms reflect the ongoing cultural discourses surrounding menstruation rather than to accept them at face value as objective medical texts.

A recent systematic review of the impact of the use of seeking medical advice from the internet on the patientphysician relationship sheds light on the positive role online medical advice can have on the patient experience. The report proposed that 'patients used the information found on the Internet to help them prepare for their visit, ask better questions, and understand what the physicians told them' (Tan and Goonawardene, 2016). This suggests that online medical advice has become a fully integrated part of contemporary healthcare, and has the potential to partially democratise the clinical encounter, providing a baseline of information common to both patient and physician. These sources are also particularly useful as they are clearly set apart from often sensationalist narratives of the media or commercially driven platforms (MacCabe and Hull, 2014). However, it is important to note that while these sources provide some information, they are quite limited and lack a recognition of the broad variety of experience of menstruation. All three examples discussed below are authored and / or medically reviewed by qualified medical doctors, but they tend not to provide reference to wider research or external information. Rather, they uphold the clinician as the primary recognised source of authority rather than acting as gateways to information that might engender potential conflict between patient and clinician in a small number of cases (Tan and Goonawardene, 2016).

\section{FRAMING MENSTRUATION IN ONLINE MEDICAL ADVICE}

All three articles are relatively short and written in an accessible style, and the analysis includes only the main text of the article. Additional content, such as advertisements - which were present on Boots WebMD and Patientinfo — and other website content were omitted from the sample. The articles varied slightly in length and format, the NHS Choices piece being shortest at 906 words over a single web page. The Boots WebMD article was presented over three click-through pages, with the main body of the text totalling 1477 words. The article available on Patientinfo is the longest of the three examples, with the main text of the article totalling 1767 words over a single page. In order to identify similarities and divergences between the three example articles, a combination of quantitative and qualitative analysis was used. This includes a thematic analysis of all results returned under the search term menstruation from the home page of each selected platform and initially coded according to the categories provided by each individual platform. Boots WebMD returned seventy-eight results, fifty-three of which were categorised as medical reference articles. The largest group of results returned by the NHS Choices search was the 'uncategorised' group, at twenty-five of the forty-seven total search results. Patient.info returned by far the greatest number of results - 475 - however the majority of these were user-generated topics from the platform's discussion forum. This user-generated content was omitted from the analysis in this article, which concentrates solely on the main authored editorial articles from each website on the topic of menstruation. However, user generated content generated a significant amount of discussion by the platforms users that could be fruitfully explored in further research. Of the remaining eighty-one results returned by the search conducted on Patient.info, sixty-four are aimed at medical professionals and the remaining seventeen at a general lay audience. Patient.info is the only website which has two defined streams of content - one for medical professionals, the other for lay readers. 
Feminist Encounters: A Journal of Critical Studies in Culture and Politics, 2(2), 15

Table 1. Frequency and percentage of coded categories found in search results for menstruation on Boots $W e b M D$, NHS Choices and Patient.info. Percentages are rounded to two decimal places, and therefore do not equal 100

\begin{tabular}{llll}
\hline THEME & Boots WebMD & NHS Choices & Patient.info \\
\hline neutral & $13(16.67 \%)$ & $4(8.51 \%)$ & $2(2.47 \%)$ \\
\hline problems & $19(24.36 \%)$ & $12(25.53 \%)$ & $10(12.35 \%)$ \\
\hline hormones \& contraceptives & $4(5.13 \%)$ & $5(10.63 \%)$ & $11(13.58 \%)$ \\
\hline menopause & $5(6.41 \%)$ & $5(10.63 \%)$ & $4(4.94 \%)$ \\
\hline pregnancy & $8(10.26 \%)$ & $9(19.15 \%)$ & $4(4.94 \%)$ \\
\hline puberty & $8(10.26 \%)$ & $4(8.51 \%)$ & 0 \\
\hline other & $21(26.92 \%)$ & $7(14.9 \%)$ & $50(61.73 \%)$ \\
\hline
\end{tabular}

This initial analysis illustrates the broad similarities and differences across the three platforms, for example it reveals that Boots WebMD is the only one that presents sponsored advertorial alongside its medically reviewed content. The majority of the content across all three websites relates to reference articles providing some form of medical information. To gain a thematic overview, the search results were coded under the following broad themes: neutral information on menstruation; problems relating to menstruation; hormones and contraception; menopause; pregnancy and related conditions; puberty or growing up; other. The search results grouped under the theme other include articles on connected but not necessarily directly related topics such as 'Headaches basics' from Boots WebMD, 'Do I have an anxiety disorder?' from NHS Choices, and 'Cold Sores' from the Patient.info website. Other is the largest thematic grouping for Boots WebMD and Patient.info, in the latter case being a greater proportion than all the other themes combined.

In two cases - Boots WebMD and NHS Choices - the next largest thematic category (after other) is problems, with Patientinfo presenting slightly more articles on the theme of hormones and contraceptives. Overall, fewer articles were coded neutral, - which includes articles that provide general information on anatomy and physical processes such as the menstrual cycle, than as problems, with the lowest number and proportion of neutral articles appearing on the Patient.info website. This is perhaps not surprising if we consider that advice is more likely to be sought to explain anomalous or negative experiences rather than to confirm a positive one. Patient.info is the only platform where I found no article titles that directly reference puberty and growing up, reflecting perhaps the medical focus of this resource, as it caters to both medical professionals and lay readers, with nearly $80 \%$ of the articles returned under the menstruation search being directed specifically towards their professional user base. The analysis of the menstruation-related content across the search results and the detailed analysis of specific articles from the three platforms suggested three key narratives through which menstruation is framed. These are: menstrual normativity ${ }^{2}$; pregnancy and menstruation as failed fertilisation within a heteronormative framework; and positive versus problematic periods. There are multiple discourses surrounding menstruation in Western culture which have overlapped and influenced each other. For example, late eighteenth and early nineteenth century medical views on menstruation were heavily informed by moralistic Christian ideologies (Lander, 1988: 10-25), and nineteenthcentury medical narratives often characterised menstruation as traumatic and debilitating (Martin, 2001: 35). In contrast, third-wave feminist activists have strongly resisted the tendency of medicalised models to view menstruation as a 'problem in need of a solution' (Bobel, 2010: 7). This is reflected in the contemporary framing of menstruation simultaneously as a biological and social phenomenon (Lander, 1988) which 'symbolizes both reproductive and sexual potential' (Lee, 1994: 360) of the menstruating body. Menstruation has only begun to emerge from a culture of silence and shame relatively recently, not helped by the sex-segregation of topics such as puberty in the classroom which means that boys (and therefore adult men) have rarely benefitted from menstrual education at school. As stated by UK Member of Parliament, Paula Sherriff, menstrual education for children of all genders is crucial as many 'boys will go on to be husbands, fathers, teachers or doctors' (House of Commons, 2017: c175WH).

In order to identify themes within each article a keyword search was conducted (see Table 2). Keywords were selected to search for established notions of menstrual norms and further keywords were added — such as childbearing and men - as they emerged from an initial reading of each of the articles. A number of keywords yielded no results in the three selected articles — these were terms relating to gender-neutral language, specifically negative terms relating to stigmatisation, and alternative menstrual products.

The analysis illustrates that while menstrual activism and contemporary feminist discourse discuss menstruation without constraining it to any single gender identity, these everyday medical texts continue to utilise a traditional binary gender framework. Within the selected articles, the words woman or women appeared 21 times [see Table 2], and girls appeared four times. The more scientific word, female, referring to biological sex was utilised infrequently - appearing most in the Patient.info article — demonstrating that while these texts have roots in medico-scientific language, their language is heavily influenced by more cultural constructions of gender. No gender-neutral terms such as menstruator appear across the material reviewed in any of the articles. This also reinforces traditional binary

2 Josefin Persdotter uses the term menstrunormativity in the forthcoming publication 'Introducing Menstrunormativity' (forthcoming 2019).

(C) 2018 by Author/s 
Table 2. Keyword frequency within the Boots WebMD, NHS Choices, and Patient.info articles on menstruation

\begin{tabular}{|c|c|c|c|c|}
\hline KEYWORD & BOOTS WEBMD & NHS CHOICES & PATIENT. INFO & TOTAL \\
\hline period(s) & 21 & 34 & 64 & 140 \\
\hline you(r) & 3 & 53 & 78 & 134 \\
\hline menstrual cycle / menstrual period & 26 & 2 & 11 & 39 \\
\hline woman / women & 14 & 7 & 9 & 30 \\
\hline pain / painful & 6 & 1 & 14 & 21 \\
\hline tampon & 3 & 10 & 8 & 21 \\
\hline pregnant / pregnancy & 8 & 7 & 5 & 20 \\
\hline normal & 11 & 0 & 7 & 18 \\
\hline change(s/d) & 3 & 3 & 9 & 15 \\
\hline $\operatorname{girl}(\mathrm{s})$ & 3 & 6 & 5 & 14 \\
\hline sanitary pad & 2 & 7 & 2 & 11 \\
\hline average & 3 & 2 & 3 & 8 \\
\hline female & 1 & 0 & 7 & 8 \\
\hline premenstrual & 1 & 3 & 3 & 7 \\
\hline abnormal / abnormalities & 0 & 1 & 4 & 5 \\
\hline menstrual cup & 0 & 4 & 1 & 5 \\
\hline vary(ing) / varies & 3 & 0 & 2 & 5 \\
\hline emotion(al) & 3 & 1 & 0 & 4 \\
\hline PMS / PMT & 0 & 4 & 0 & 4 \\
\hline baby & 3 & 0 & 0 & 3 \\
\hline typical(ly) & 2 & 1 & 0 & 3 \\
\hline childbearing & 1 & 0 & 0 & 1 \\
\hline dirty & 0 & 0 & 1 & 1 \\
\hline man / men & 1 & 0 & 0 & 1 \\
\hline mood & 0 & 1 & 0 & 1 \\
\hline childbirth & 1 & 0 & 0 & 1 \\
\hline
\end{tabular}

understandings of gender that codifies menstruation as a strictly biological phenomenon that happens to cis women, and leaving non-binary and/or trans menstruators out of discussion. It is interesting to note that while none of the articles contain words that explicitly reference stigma, no explicitly positive words are used to describe menstruation either. The articles reinforce a narrative of menstruation that is personal and private, referring only to medical professionals — or a man who might provide sperm to fertilise an egg — other than the reader, assumed to be a woman or girl.

\section{MENSTRUAL NORMATIVITY}

Emily Martin (2001) contrasts prevailing medical metaphors used to describe menstruation, pregnancy and menopause in terms of mechanical production with the oral testimonies of the lived experience of these phenomena of women from different class and racial backgrounds. She states that 'in the current medical model, regular periodicity between well-defined limits is considered normal' (Martin, 2001: xi), and that while 'regularity is normal, good, and valued; irregularity is abnormal and negatively valued' (ibid). She goes on to draw on scholarship from heart health and epidemiological research which suggests that increased flexibility and variance might actually suggest a greater ability to adapt to the various stresses of life such as changing environments or life circumstances (Martin, 2001: xii). The influence of medicine on broader understandings of menstruation is underscored in Lander's analysis of menstruation as 'simultaneously a biological event and a cultural event' (Lander, 1988: 9) which has often been presented to women through the lens of 'medical ideology, reflecting social ideology' (ibid). The cultural representation of menstruation has been explored in different forms, with researchers exploring areas from menstrual activism (Bobel, 2010), to art and literature (Bobel and Kissling, 2011), to print culture and advertising (Houppert, 2000; Røstvik, 2018). What emerges from this body of literature on the subject of menstruation and the many other recorded accounts of the phenomenological experience of menstruation from people who menstruate, that menstruation is a highly individual and varied experience. From the physical experience of menstruating through to attitudes informed by the differing intersections of class, gender, culture, racial and socio-economic identity, for all the physiological things menstruators might have in common, there are many other aspects — physical and social — that might differ considerably.

Of the three selected main articles on menstruation, Boots WebMD uses the term normal most frequently (11 times in total), while the NHS Choices article does not use it at all. The Boots WebMD article is titled 'Normal menstrual cycle and periods' (Boots WebMD, 2016: 1), with the term repeated in the first subtitle: 'what happened during a normal menstrual cycle?' (ibid). The doubling of the term normal at the opening of the article reinforces 
the notion of a normative menstrual experience, which in this particular text is presented almost exclusively in relation to pregnancy:

During a woman's childbearing years, her body will usually experience a menstrual cycle: a complicated cycle controlled by hormones to prepare her body for pregnancy. (Boots WebMD, 2016: 1)

The first sentence of the article firmly situates menstruation in a context of 'childbearing' and 'pregnancy', alienating those menstruators who do not associate their menstrual cycle and flow with a desire — or are unable - to become pregnant. The sentence also constructs a dichotomy between the menstruating person and their body. The subject - in this instance the woman — and her body are alienated by a process that is described as controlled and experienced outside the menstruator. The rest of the article echoes this oddly detached language referring to 'a woman' or 'a girl' and 'her body', or to the reproductive organs and gametes as though they are independent entities instead of parts of one whole. This reinforces the notion that the process of menstruating is something unpleasant and unnatural that should be kept at arm's length. The framing here contrasts starkly with both the NHS Choices and Patient.info articles which both use the terms you/yours frequently, and develop a more individualised, and perhaps patient-conscious tone and description of menstrual experience.

Instead of describing or qualifying menstruation itself as something with a normal standard, Patient.info refers to 'the common variations which are normal' (Patient.info, 2017), as well as 'some common problems' (ibid). This is a small but significant difference in the conceptualisation of menstruation presented across the three examples: instead of establishing a description of menstruation that is itself normative, variation is framed as the norm. It is especially important that sources of information with some perceived form of medical authority are careful to establish variation as a key feature of menstruation, and to clearly define ranges of expected experience that are not considered to be pathological. In contrast, Boots WebMD cites a vague cycle length of 'around 28 days' (Boots WebMD, 2016: 1) which 'varies from woman to woman' (ibid). None of the advice provided across the three articles establishes clearly how much variation outside twenty-eight days a menstruator might experience before needing to seek further advice from a primary care provider, other than to suggest they should take a pregnancy test. As Rees notes, in socio-cultural terms, 'the tyranny of the notion of a bodily norm means that, in the act of being human, our very humanity — our age, weight, ability — sets us up to fail' (Rees, 2017: 7). In my experience this is no less true for menstruation: it is an event that varies greatly between — and for — individuals, but has often been framed as an unchanging, regularly cyclical and predictable event.

\section{PREGNANCY AND MENSTRUATION AS FAILED FERTILISATION}

Fertility and reproduction are central to contemporary Western debates on gender, and as Cordelia Fine observes, we are 'spellbound' (Fine, 2017: 14) by debates surrounding hormones and the contested ground of reproductive evolutionary divergences between the two traditionally recognised biological sexes (ibid). Menstruation — and its absence — are powerful signs and sources of anxiety for people who are trying to conceive, or are worried they may be facing an unwanted pregnancy. Despite my own experience of severe dysmenorrhoea ${ }^{3}$, there have been times when the arrival of my period has been a huge relief. I can only begin to imagine the depth of grief that might accompany starting your period if you are hoping to become pregnant. While the absence of menstruation due to pregnancy may be good news for some, and it is entirely reasonable to discuss fertility in the context of the menstrual cycle, it is also important to consider that not all people who menstruate want to become pregnant at all. For example, a report by NHS Digital states that $79 \%$ of women attending Sexual and Reproductive Health Services (SRH) in England in 2016-17 were visiting to start or maintain contraception methods (NHS Digital, 2017: 10). Additionally, use of Long Acting Reversible Contraceptives increased from 18\% in 2002-3 to 31\% of women accessing SRHs in England (Health and Social Care Information Centre, 2014: 6). Martin's (2001) examination of the language of medical textbooks revealed that many described menstruation negatively as the result of a failed fertilisation process. It is striking that over thirty years later this narrative is still evident in the way Boots WebMD frames its discussion of menstruation overwhelmingly through the lens of 'childbearing' (Boots WebMD, 2016: 1).

To frame menstruation entirely through a reproductive lens assumes that the menstruator desires pregnancy, and reduces the potential of their bodies to be and be seen only as vessels for carrying babies. As Janet Lee notes, the onset of menstruation 'signifies both emerging sexual availability and reproductive potential' (Lee 1994: 344) which in a Western patriarchal society is often understood through heteronormative desires. This perspective designates the post-menarche body as reaching adulthood, and therefore ready to fulfil the dual roles of object of sexual desire and mother (e.g. Lee, 1994; Haug et al., 1987; Young, 1990).

${ }^{3}$ Dysmenorrhoea is the medical term for period pain.

(C) 2018 by Author/s 
Of the three articles analysed, Boots WebMD presents a model of menstruation that emphasises pregnancy. Words relating to pregnancy are used more frequently in the Boots WebMD article (see Table 2) than either of the other texts, reinforcing the sexualisation of menstruators within a heteronormative narrative of sex for reproduction. Patient.info and NHS Choices briefly discuss pregnancy within the broader context of the menstrual cycle, foregrounding individual experience and cyclicity rather than fertility, going some way towards decentring traditional gendered childbearing roles and heteronormative expectations from the cultural construction and understanding of menstruation.

Patient.info frames menstruation as a cycle of hormonally mediated bodily changes. It makes very little mention of pregnancy at all until its explanation of the phases of the menstrual cycle, noting that it is possible to become pregnant if 'you have recently had sex and there are sperm in your womb' (Patient.info, 2017) during the first half of the menstrual cycle. NHS Choices takes a similar approach and tone, restricting the discussion of pregnancy to a discrete section within the article, and places fertility within the broader context of the menstrual cycle and contraception through providing links to these topics. Both of these platforms describe menstruation as an internally regulated process that is affected by fluctuating hormones within the individual menstruator.

In contrast, Boots WebMD presents menstruation in terms of not becoming pregnant. The article presents an unnecessary tautology of a 'man's sperm' (Boots WebMD, 2016: 2) fertilising the passive egg, noting that if that does not happen then menstruation begins. Instead of emphasising menstruation as a cyclic process, it presents the interaction of an external factor as the core agent of influence in the process. Patientinfo and NHS Choices discuss pregnancy in a comparatively clinical and neutral tone, and while both mention sperm, neither of these articles mention men. While their discussion of sexual intercourse and pregnancy draws on scientific definitions of biological sex in the context of reproduction, they do not explicitly frame this within a heteronormative narrative of sex to the same extent that Boots WebMD does.

Furthermore, the topic of pregnancy is threaded throughout the Boots WebMD article, bookending the advice from the first paragraph to the final section sensitively titled 'How many eggs does a woman have?' (Boots WebMD, 2016: 3). While all three examples contain sections that address related conditions and problems which might impact the menstrual cycle, Boots WebMD names pregnancy first and foremost (ibid). This may be true in the sense that being pregnant stops periods, but there are many other factors that impact the menstrual cycle. The article does list other factors, but only after a short paragraph detailing pregnancy as a separate and overriding condition. Even in the list of other conditions that might affect menstruation, emotional stress is linked directly to pregnancy - even though it seems reasonable to assume that any number of different areas in the life of anyone that menstruates could induce stress. It demonstrates the depth of the investment in the model of menstruation as failed pregnancy, and the overarching outdated and sexist model which emphasises the woman-as-mother role, defined by heterosexual relationships and a pre-supposed normative desire to produce babies.

This approach seems out-of-step with changing contemporary cultural attitudes towards childbearing. For example, recent data from the UK's Office for National Statistics (ONS) reveals three significant trends: in England and Wales, fewer children are being born than in previous generations; more women are delaying their pregnancies until later than they did in previous generations; and the number of women remaining childless is also rising (ONS, 2017). By foregrounding pregnancy as the most central issue relating to menstruation consistently through the advice, the proprietors of Boots $W e b M D$ seem clearly invested in a traditional ideological view of women in society, with the female body bounded by a rigid definition as a reproductive vessel.

The framing of menstruation as failed pregnancy is alienating to cis women who do not want to have children. It is also an insensitive and unnecessary reminder to those who would like to become pregnant but cannot, especially as it draws on fertility anxieties, detailing in the closing of the article that 'the vast majority of eggs within the ovaries steadily die' (Boots WebMD, 2016: 3). It is also alienating to cis and trans and/or non-binary menstruators who experience physical and/or psychological difficulties relating to menstruation and further reinforces traditional binary gender roles. For example, a poem by menstrual activist Cass Bliss Clemmer illustrates this poignantly. The piece, posted to their recently launched website, Bleeding While Trans, describes menstrual bleeding as experiencing 'another day I shed my gender' (Clemmer, 2018).

Highly gendered and binary representations of menstruation are exclusionary, forcing the definition of women as menstruators, and menstruators as women in a rigid and damaging way. To continue to frame menstruation in the terms of failed reproduction is both a failure to recognise that cultural norms have started to shift away from the patriarchal binaries of gender, and is a stubborn continuation of traditional roles that represent a diminishing section of culture. That two of the three cases studied are able to discuss menstruation without emphasising pregnancy suggests that there may be an ideological agenda at play in the Boots WebMD resource. This might be fruitfully explored further in light of the recent and ongoing campaign from the British Pregnancy Advisory Service (BPAS), which has lobbied the senior management of Boots to reduce the price of emergency contraception in line with other retailers (BPAS, 2017). 


\section{POSITIVE VERSUS PROBLEMATIC PERIODS}

As well as constructing and reflecting notions of menstrual normativity to different degrees, the articles present menstruation as positive or problematic in varying ways. The overall framing of menstruation across all three articles is overwhelmingly as problematic, with only one of the articles making any positive statement about menstruation. Menstruation is discussed in each of the texts as an experience that has to be coped with in two key areas: dealing with unwanted physical and emotional side-effects and the management of menstrual flow.

Patientinfo provides the closest thing to a positive statement regarding menstruation in all three examples, answering its heading that questions whether there is a need to change your behaviour while bleeding:

No. Carry on as normal. If you find the periods painful, regular exercise sometimes helps. Periods are not dirty; they are a normal part of a woman's life. You can go swimming, have a bath, etc. You may prefer to use tampons if you enjoy swimming. (Patient.info, 2017)

To state that menstruating is not unclean falls quite far short of positive affirmation, but to go even go this far is more than we see in the other selected texts. The enduring influence of conservative Christian values on UK menstrual culture is reflected in the continued encouragement of modesty and silence to ensure that menstruation remains hidden. While menstruators have been segregated from their communities (or have possibly chosen to be sequestered away during menstruation) across many religions and societies, Victoria Newton observes that in a contemporary UK context 'we have witnessed a historical shift from the invisible menstruating woman to invisible menstruation' (Newton, 2016: 183). The hesitant positivity presented by Patient.info is barely a cause for celebration, the rest of the paragraph is far from innocuous. Directing menstruators to exercise their pain away and to 'carry on as normal' is paternalistic and dismissive of both the deeply ingrained shame that accompanies menstruating, as well as the reality that according to the National Institute for Health and Care Excellence (NICE) anywhere between 50 and 90 per cent of menstruators in the UK are affected by dysmenorrhoea (NICE, 2014). Beyond the prevalence of dysmenorrhoea among menstruators, this instruction towards stoicism falls within the gender biases that are well documented across different areas of medicine (Holdcroft, 2007; Hamberg, 2008; Samulowitz et al., 2018). The research in this area points towards a culture of disbelief of women's pain and their ability to report their own experiences accurately (for example see Fassler, 2015; Fillingim et al., 2009; Hoffmann and Tarzian, 2008).

Simple changes to the framing of menstruation at points in the text such as this could be made to acknowledge the broad variety of menstrual experience and menstruating individuals. For example, stating that exercise and painkillers can provide relief or instead of ring-fencing menstruation as being a normal part of women's lives, it would be far more inclusive to state they are a normal part of life. This would include trans and/or non-binary menstruators, as well as acknowledging that menstruation is part of life for everybody to some degree — even people who do not menstruate might have family members, partners, or friends that do menstruate and it should be framed and promoted as a normal part of their lives too.

Menstrual management is discussed in all three texts. On the one hand these sections provide (limited) practical information on what to do while bleeding. On the other hand, other than the single example noted above the articles frame menstruation as something unclean that needs to be cleaned up and disposed of, particularly through the use of the term sanitary rather than menstrual products in the Patient.info and NHS Choices articles. Feminist scholars and activists have criticised the term feminine hygiene product (e.g. Fahs, 2016; Quint, 2017), though sanitary product seems to have elicited less robust critique. As Breanne Fahs states:

The phrase feminine hygiene [original emphasis] implies "products to keep the unkempt, unruly, unhygienic, dirty, unsanitary, bloody vagina in check" rather than simply stating the actual terms for what women use. (Fahs, 2016: 48)

While sanitary product avoids 'needlessly gender[ing]' (ibid) products as the term feminine bygiene product does, it still maintains the notion that menstruation - and therefore people who are menstruating — are fundamentally unclean. The term sanitary product also continues to shroud menstruation in euphemism, reinforcing the 'private, personal nature of bleeding' (Houppert, 2000: 81). While Patient.info and NHS Choices do at least provide subsections specifically addressing menstrual products - even if the terminology used is problematic - the Boots WebMD article provides no general practical advice for what to do while bleeding.

Perhaps most perplexing — and worrying — is that menstrual management is only mentioned by the Boots WebMD article in the context of problems relating to menstruation. Instead of providing practical advice or menstrual product suggestions, the article only refers to tampons in relation to Toxic Shock Syndrome (TSS). Furthermore, while alerting readers to the danger of contracting TSS additional information, explanation or context is subtly signposted by an embedded text hyperlink. This reflects the lasting cultural association between tampon 
use and TSS following the emergence of menstrual toxic shock syndrome as 'a public health threat to women of reproductive age' (Hajjeh, Reingold et al., 1999: 807) following the full commercial launch of the super-absorbent Rely tampon developed (and later discontinued) by Procter and Gamble (Bobel, 2010: 53; Houppert, 2000: 26). However following the introduction of guidance for proper use of tampons and changes to the manufacturing of tampons, cases of TSS have greatly reduced and now most UK cases of TSS are not related to menstruation (Sharma et al., 2018). Boots WebMD only otherwise mentions menstrual pads and tampons in relation to using a large number of pads for a heavy flow. Both of these instances provide very little practical everyday advice, and without further research from the reader could potentially cause considerable alarm. It is particularly irksome to find that an article supposedly about menstruation provides much more information and advice on pregnancy and fertility than it does how to manage periods on a day-to-day basis.

The types of menstrual products mentioned in each article are largely restricted to disposable, commercial products. This reflects the significant influence — including promoting and upholding menstrual taboo — major brands have had over menstrual management (Røstvik, 2018) and menstrual education (Houppert, 2000: 60-74). All three articles mention pads and tampons. The only alternative, reusable method suggested — menstrual cups - is noted by Patientinfo and NHS Choices. None of the articles mention products such as period underwear or reusable cloth pads and none enter into any discussion of the positive and negative aspects - such as personal comfort, initial or long-term cost, or environmental considerations - of the methods mentioned other than how they collect or absorb the blood or that tampons might be 'more convenient' (Patient.info, 2017). This is another area where cis-normativity might be productively countered through basic information, as menstrual products have emerged in recent years - such as absorbent boxer shorts - that break gender binaries and the gendered expectations that are deeply ingrained in the use of and marketing of traditional menstrual products. Nonconventional products such as menstrual cups or absorbent period underwear in a variety of styles other than traditionally feminine styles - are crucial for trans and/or non-binary menstruators who might be at risk of violence if found to be menstruating, as Fahs describes the experience of one of her psychotherapy clients:

Nash spent his entire menstrual cycle hoping not to bleed through, and he constantly feared that other men would hurt or even kill him if they discovered him as FTM [female-to-male]. Menstruation felt like a lethal form of "outing" him as a trans man. (Fahs, 2016: 82)

Providing a greater range of information is crucial to enable all menstruators to find methods that are most suitable to their individual situation - from environmental or health concerns, to serious issues surrounding personal safety - and alternatives to disposable menstrual products should be more comprehensively incorporated into medical advice and information resources.

The final aspect of the three articles to compare is the information presented on the physical and emotional symptoms associated with premenstrual syndrome (PMS) and other problems such as painful, irregular, heavy or absent periods. The quantitative analysis suggests that Patient.info places most emphasis on physical symptoms of PMS; it has by far the most instances of the word pain (see Table 2). However, a close comparative reading of the three articles provides a more nuanced picture of their presentation of negative premenstrual symptoms.

Patient.info and NHS Choices provide links to further information on PMS whereas Boots WebMD does not, linking only to general pages relating to some premenstrual symptoms — such as bloating, depression and headaches - rather than providing further information in the specific context of the menstrual cycle. Boots WebMD lists the most premenstrual symptoms of all three articles — ten in total — including a broader range of emotional and physical complaints, such as trouble sleeping or concentrating and feeling upset or depressed. The broader list of potential negative premenstrual symptoms presented is one of the most positive aspects of the Boots WebMD article. Listing a range of symptoms, rather than hiding most behind a click-through link, gives a more detailed and accessible understanding for the reader and normalises a broader range of symptoms overall. It is also worth noting that Boots WebMD is the only of the three articles to refer to premenstrual symptoms, rather than using the medical terms PMS, PMT (premenstrual tension) and PMD (premenstrual disorder). This perhaps reflects a more individualised and emotive approach — also expressed in the framing of menstruation around pregnancy — by this resource, in contrast to the more straightforwardly medical tone and content presented by Patient.info and NHS Choices which are both directly associated with the medical profession in the UK.

Amongst the description of negative aspects of menstruation the Patient.info article notes that 'you may feel irritable before a period' (Patient.info, 2017), that such symptoms are 'normal' (ibid) and that if you experience more 'severe' (ibid) symptoms then you may be experiencing PMS. There is no indication of what constitutes a normal or severe level of discomfort, which on the one hand may encourage menstruators to consider what is normal for them, but on the other provides no frame of reference to suggest what sort of pain should warrant further investigation. Moreover, the only emotional impact mentioned is that you might 'feel irritable' (ibid). This is a vague and reductive description which plays into the stereotypical and stigmatising view of premenstrual people as potentially 'violent, irrational, emotionally labile, out-of-control, and physically or mentally ill' Johnston- 
Robledo and Chrisler, 2011). Adverse physical symptoms such as period pain and heavy periods are afforded more detailed and reassuring advice, reiterating that experiences vary: 'some women have more pain that others' (Patient.info, 2017). The weighting of the advice towards physical symptoms reflects ongoing public, political e.g. the equality4mentalhealth.uk campaign launched in 2015 (Wax, 2016) — and clinical debates (Millard and Wessely, 2014) surrounding the lack of parity between physical and mental health care in the UK.

NHS Choices places very little emphasis on menstrual problems such as pain and excessive bleeding, instead providing links to these related conditions at the end of the first section of the article. Unlike the other examples, which frame periods in terms of related problems or through establishing normal menstruation in their titles, the NHS Choices article is neutrally titled as 'Periods'. This neutral framing is carried through the article, which though quite generalised and brief provides ample links to related pages and notes that menstruation is a varied experience. The article neither dwells on nor dismisses unwelcome physical conditions such as dysmenorrhea, and along with the neutral title this potentially creates a more neutral space for readers to construct their own meanings around menstruation. While possible related physical conditions are given little attention, PMS is afforded its own subheaded section. This provides a link to a more detailed page, and while it adds 'mood swings' (NHS Choices, 2016) to the list of possible symptoms, the only other emotional affect named is 'feeling irritable' and 'loss of interest in sex' (ibid). While a much broader range of symptoms appears on the linked page on PMS, it would it would be useful to include a broader range of possible emotional symptoms within the main article in order to reassure those affected and promote a more nuanced understanding of PMS overall.

In the first part of the article, I have explored the largely problematic ways in which menstruation is framed in a selection of everyday medical texts published online and how this might inform and / or reflect contemporary understandings of menstruation. I will now discuss the practice-led research that was the main catalyst for the above analysis, and my use of performative artistic techniques to develop alternative personal understandings of my own experience of menstruation.

\section{DECONSTRUCTING MENSTRUAL STIGMA THROUGH ENTANGLED AUTOBIOGRAPHICAL ART PRACTICE}

My artmaking is an exploration of the continued rippling effect of encountering menstruation — both my own and the representation of menstruation generally — stuck between medicalisation and the languages of advertising built upon maintaining the secrecy of the event of menstruation. As Jennifer Weiss-Wolf observes, to sell menstrual products for much of their history 'the message was always the same. No leaks, no stains. No pain. No problem' (Weiss-Wolf, 2017: 14). Against a backdrop of pervasive cultural messaging, the experience of menstruating has become aestheticised, commodified, and open to comparison with the normative experiences portrayed, however caricatured they may be. My work joins others who present menstruation on their own terms, as a form of menstrual activism which Bobel and Kissling describe as revealing:

how women internalize destructive messages about womanhood including notions of our bodies as messy, unruly things (yes, things) that need to be tidied up, medicated, plucked, smoothed, and trimmed. (Bobel and Kissling, 2011: 123).

It is perhaps not stated often enough that these messages are not only internalised by cis women — they are available to and internalised by everyone, giving rise to anxiety for those who menstruate and reinforcing harmful, misogynistic expectations of female bodies in others. Chris Bobel (2007: 87) describes menstrual activism as growing from the 1970s women's health, environmental and punk movements with 'a scathing critique of the dominant Western cultural narrative of menstruation, resisting the framing of menstruation as dirty, shameful, and something best hidden' (ibid), and to challenge the binary view of gender that 'equates menstruation with womanhood' (Bobel, 2006: 89). Bobel has also noted that the broad range of activities undertaken over decades 'reveal[s] the diverse ways menstrual activism has responded to shifting temporal, political, and social contexts' (Bobel, 2008: 739). Fahs (2016: 96-97) outlines in detail the important work menstrual activists have undertaken to challenge the proliferation of commercial menstrual products, resist the pathologisation of menstruation, and encourage more positive relationships to bleeding for menstruators and non-menstruators alike. She also points to different strategies that could be adopted by menstrual activists that go beyond the traditional focus on commercial menstrual products (Fahs, 2016: 104). These strategies include 'rebelling against the culture of secrecy and shame around menstruation' (ibid) and 'adopting radical postures of “outing" oneself as menstruating women' (ibid).

In my visual artwork, I deploy my menstruating body in a performative gesture that both 'outs' me as a menstruator and illustrates a menstrual cycle that does not fit the normal descriptors provided by medical texts, such as those analysed above. Cycles (2016-2017) (see Figure 1) is a series of 3 metre long hand stitched scrolls, each divided into 28 sections - sometimes a few more or less — by a knotted red stitch. I began the work as a 


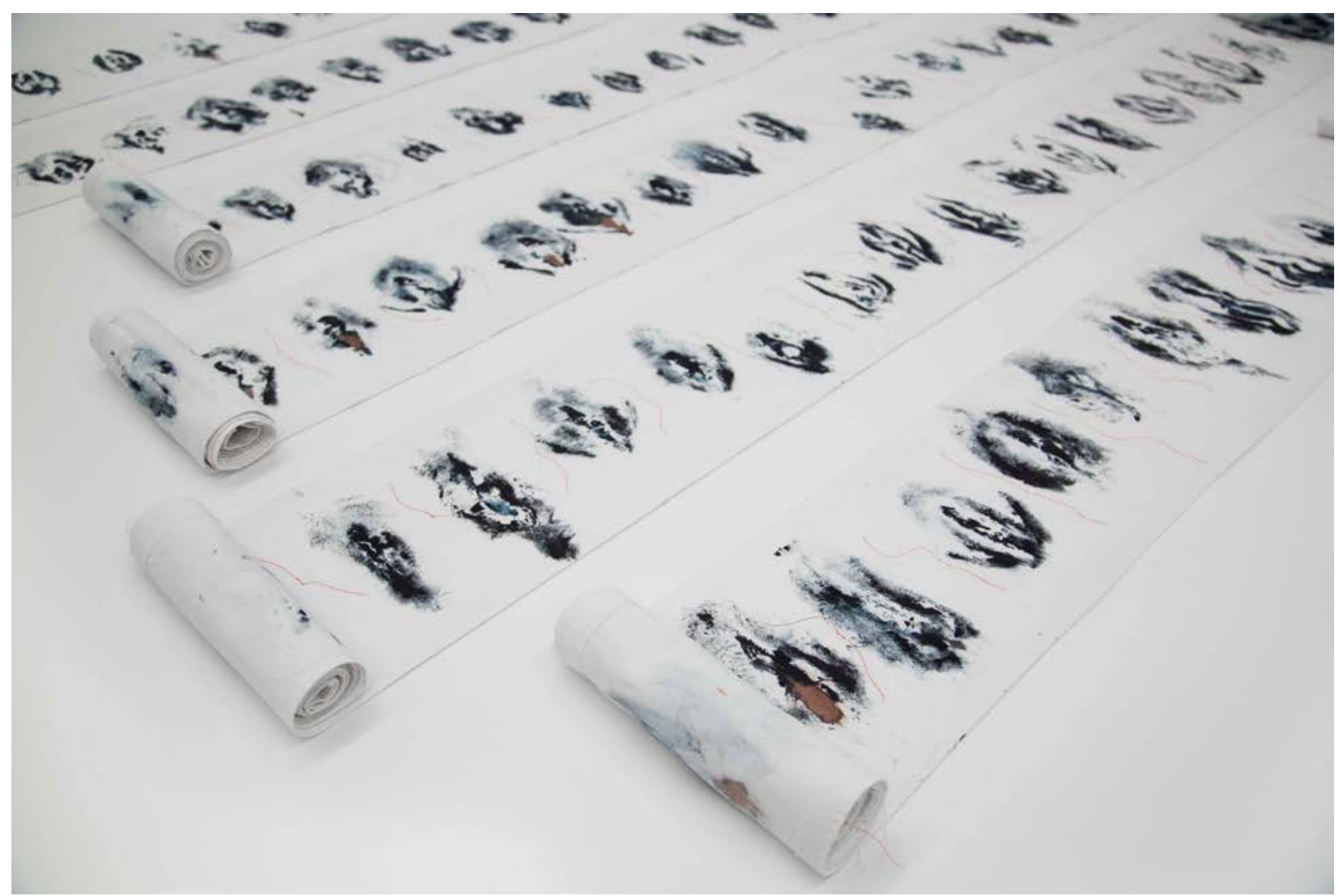

Figure 1. Bee Hughes, Cycles, 2016-2017. Acrylic and menstrual fluid on hand stitched linen scrolls, installation size variable

way to confront my painful, inconsistent menstrual cycle, making a single print each evening over a six month period by applying paint to my vulva with a small brush or my fingers. Once inked, I carefully press the linen against my vulva, transferring the paint and any body fluids present to the cloth. I refer to my practice as performative printmaking, as it goes beyond traditional printmaking techniques that utilise mechanical apparatus to create images. Instead, I utilise my body as printing plate and press, with the resulting serialised images forming a material document of the gesture. The artworks form a documented performance of my irregular and changing menstrual cycle, a direct and public challenge to the 'stigma of menstruation' (Johnston-Robledo and Chrisler, 2011: 9).

The white scrolls with carefully divided sections are intended to evoke notions of the sanitary, suggesting both the clinical setting and the private sphere: white sheets; white pads and tampons; white cotton knickers; the dread of staining any of them. In her analysis of menstrual stains in relation to Kristeva's (1982) exploration on the abject, Fah's notes that if a menstruator has bled through their clothing, they have 'bled through [original emphasis] not only their literal underwear and pants but also transformed the boundary between public/private, self/other, and animal/human' (Fahs, 2016: 38). The ink and blood left on the scrolls are a direct retort to a persistent 'emphasis on the private, personal nature of bleeding' (Houppert, 2000: 81) and a standardised rather than individualised idea of the menstrual cycle. Furthermore, in exposing the variations in my own cycle through the Cycles prints, alongside my other works in poetry and in talking openly about my periods in relation to these works, I aim to counter this normalised period and present a personalised account of a menstruating body. The reiterative process of making the prints, and the repetition in my other works, are my attempt - to paraphrase Sara Ahmed — of not reproducing the grammar of patriarchy (2017: 4) which underpins the narrative of menstrual taboo. Judith Butler refers to 'a recurrent moment [...] when we grasp that we are in the midst of reiterating a norm, even that a norm has entered into a basic sense of who we are, and start to deviate [...] from that more obedient sense of repetition.' (Butler in Ahmed 2016: 484). My artistic practice begins with and stays with a realisation that for most of my adolescent and adult life I too had been reiterating the norm of period taboos.

To make these works is to fall out of alignment — to borrow Ahmed's term (2017:55) — with what is expected: silence, shame and deference to taboo and to recalibrate the way in which I act out my period, both at a personal level and as an artist facing an outside audience.

At the core of the Cycles project is a desire to de-mystify menstruation and bodies that menstruate as distinct from the norm, as a phenomenon that affects most of the population in some way — directly as menstruators, or indirectly as the partners, friends or relatives of menstruators. That vulvas and vaginas - and images of them exist outside the erotic, the sensational, the medical and the abject. I aim to present the everyday-ness of 
a period is

a period is the menstrual cycle

menstrual cycle

a period is the

cycle

cycle

a period is

is a woman bleeds

when a woman bleeds from her vagina

woman bleeds

bleeds her vagina

bleeds

bleeds

bleeds period. a woman

a woman

Figure 2. Bee Hughes, a period is..., 2016, visual poem

menstruation, and suggest the ever-changing and fluctuating nature of the human body as no two vulva prints are the same. These works are at once intensely personal and present a fragmented and incomplete picture of myself as the artist, which can only be understood within my biography with access to further discussion, gallery presentation, additional writings such as this article, or oral presentations. Though my work presents an alternative image of menstruation, when read alongside the online medical advice, it becomes apparent that neither art nor medicine can provide a complete picture alone. Both medical and 'vernacular knowledge and belief' (Newton, 2016: 1) which inform the overarching cultural constructions of menstruation outlined above are incomplete. Reading cultural, medical and artistic articulations through each other offers the potential to entangle everyday 'folk' (Newton, 2016) meanings, medical knowledge and personal experience towards surfacing empowered, nuanced understandings of menstruating bodies made accessible through the dissemination of artworks and autobiographical context in multiple forms.

Alongside and intertwined with the visual work are poetic experiments which began as written and visual poems, later developed into soundworks. My poems are composed by cutting-up or redacting and permuting phrases from online advice pages — such as those analysed above - and stem from a desire to explore my experience of not feeling accounted for and listened to in my ongoing encounters with medical professionals regarding my own menstruation. In the tradition of cut-up writing, the source text is re-appropriated into a radically different context. Miles writes of William Burroughs (one of the earliest and most well-known cut-up writers) and his analogy of language as a virus that the only way neutralise the power of those in control 'is to destroy their means of control: their language' (Miles, 2010: 126). While the poems are collaged from found words that are themselves divorced from any single subjective experience, they hold an emotional resonance. Through permuting and repeating phrases and words, I attempt to recreate the feeling of searching for medical advice online, skimming texts and finding little reassurance. The poem a period is... (2016) appropriates text from the NHS Choices website, intertwining words of medical authority with personal experience and emotion. This intervention aims to deconstruct and complicate the language of menstruation I found while consulting everyday medical resources online. I enmesh my embodied experience with these texts as a personal means of questioning and reconfiguring the way menstruation and menstruating bodies are understood by moving the text from its assumed original context as medical to a more explicitly cultural space. Through these experiments there is a potential to highlight the complex entanglement of culture and medicine in the understanding of menstruation that has been so wellarticulated by many of the scholars cited here. Through exposing and entangling the medical with the cultural in this way, this research aims to underscore the extra-clinical space of online medical advice as part of everyday experience, as a cultural medical hybrid text rather than a strictly specialist part medical authority that is traditionally inaccessible to lay audiences. 


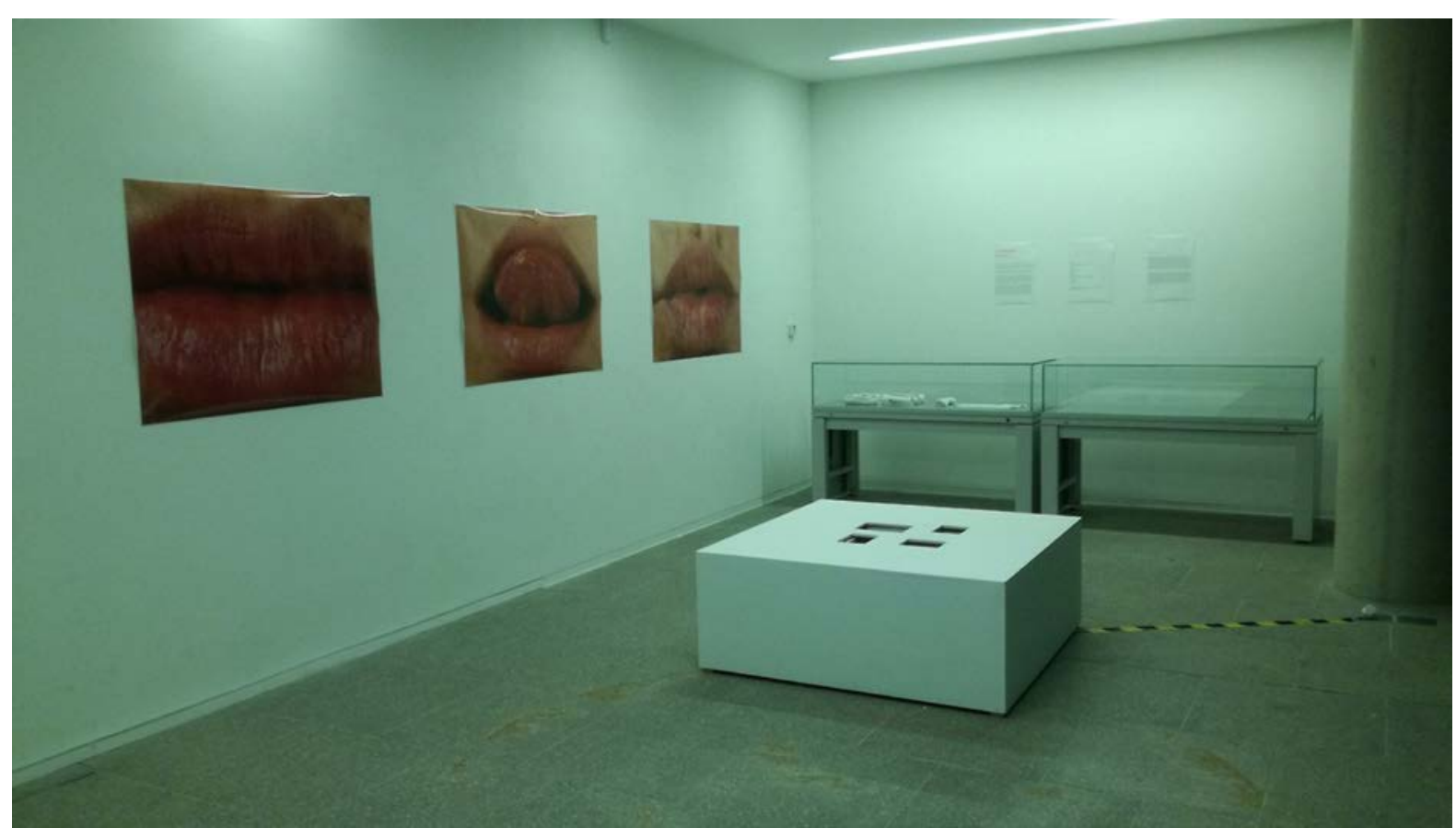

Figure 3. Installation image of Comfort Zones exhibition at Liverpool School of Art \& Design, 2017. Photograph: Bee Hughes. Pictured: Eva Petersen, Yes, I am Looking Straight at You, 2017, photographic prints. Bee Hughes, Infinite Cycles, 2017, multimedia installation.

In the poem I aim to mirror the feeling of frustration that arose while reading online advice which presents little space for variation, outliers or non-conforming bodies and cycles. For example, in a period is... (2016), the repetition of $a$ woman and bleeds reflects irritation that the original text described bleeding categorically as something women do. The exasperation was twofold: first, there was the implication that womanhood should be defined by the ability to menstruate; second was the idea that only cis women menstruated. In the reiteration and fragmentation of these phrases, I am opening this orthodoxy up to question rather than reinforcing it as hard fact — by repeating the words until they lose their meaning and their power to define menstrual experience.

This sentiment carried through into my poems as they are translated into soundworks. This process of returning the words to the body through my voice reflects the performative form of the visual works. Un-voiced (Hughes and Petersen, 2017a) combines a spoken word poem composed by redacting the same advice used for a period is...(2016) with non-vocal breathing sounds composed and performed by Eva Petersen. The piece plays on the expectations of a performance, and the expectation of silence that surrounds the social stigmatisation of menstruation. Petersen is a vocal artist whose entire performance for the piece is comprised of pre-vocal breathing exercises. In contrast, a clear voice dispassionately reads words pulled from the medical text: the pronouns used in the text — you, woman, girl — and the words period and vagina emerge from the breathing. The tension between the intimate breathing sounds and the disjointed, context-free words alludes to the various ways contemporary culture tends to silence or homogenise individual experiences. When played on a loop - as in the collaborative exhibition Comfort Zones (Hughes and Petersen, 2017b), alongside close-up self-portraits of Petersen's mouth taken whilst singing and the Cycles prints - the repetitive, fragmented phrases echo (sonically as well as metaphorically) the growing sense of frustration associated with my ongoing exploration of everyday medical texts and traditional ideologies and attitudes surrounding menstruation.

\section{CONCLUSIONS}

My artistic practice is a work-in-progress which will continue to develop and respond as this research evolves. Preliminary findings of this practice-led research have enabled me to situate my practice as alongside menstrual activism, as it continues to evolve as a method through which to question and challenge socio-cultural stigma and normative assumptions constructed through sources of medical and cultural authority. My practice has become a space of personal resistance to normative constructions of menstruation, and through its dissemination I hope to contribute to ongoing discussions that erode the norm of menstrual taboo. In the re-appropriation of medical texts and re-embodiment of my work through sound — as in Un-Voiced (2017) —I aim to continue to develop my own voice and understanding of menstruation, particularly investigating sites of tension where my own work might further deconstruct or become complicit in normative ideas and practices of menstruation in wider culture. 
Depicting menstruation that conforms to neither contemporary ideals - the regular or the regulated — as an everyday rather than pathologised occurrence, complicates the traditionally assumed binaries of 'health/disease, whole/broken, normal/abnormal' (Price and Shildrick, 1998: 4) often attached to biomedicine. Though these binaries have been eroded through the turn towards a reinstatement of the 'materiality of the body' (ibid: 7) in feminist critical theory, this turn has also been criticised for remaining 'highly abstract' (ibid). The practice of artmaking, particularly autobiographically entangled, visceral and phenomenologically produced through the body has the potential to foreground — literally and figuratively — 'the material body in the very acts of academic production' (ibid) and anchor theoretical explorations in tangible, relatable situations.

This developing body of work began as a highly personal method of claiming control over my irregular menstrual cycle. It has shifted towards what may be described as an artistic practice not developed 'in service or opposition to the clinical and life sciences, but as productively entangled with a "biomedical culture" (Viney et al., 2005: 2). It is produced in the context of a broadened notion of the medical 'beyond the primal scene of the clinical encounter' (ibid) through engagement with online medical texts utilised outside (as well as within, in some cases) the clinic. Cycles presents an embodied experience that falls between and outside the intertwined medical and vernacular ideals of menstruation. Modified by hormonal contraceptives, my body presents neither a normative ideal 28-day cycle reinforced by now widespread use of the contraceptive pill (Oudshoorn, 1994) — nor fulfils the further promise of a cycle fully under control and rendered invisible through the cessation of menstrual flow achieved through a form of 'technological manipulation' (Balsamo, 1996) provided by hormonal contraceptive developments.

My analysis of the examples websites reflect that these examples of health advice remain grounded in traditional binary conceptions of gender and focus specifically on menstruation as a phenomenon that happens to cis women. Boots WebMD, NHS Choices and Patient.info present menstruation in relation to problems, yet offer limited practical advice on managing or coping with the experience of bleeding and any other related symptoms. I found limited positive framing of menstruation in the suggestion across all three studied sources that variation between individual experiences is normal. However, there remains an emphasis on defining and reinforcing normative notions of menstruation, and a limited potential for democratising medical knowledge between patients and physicians. Instead of providing a clear measure by which to understand whether your experience is medically normal, the construction of menstrual normativity has obscured, ignored or minimised difficulties experienced by nonnormative menstruators.

Framing menstruation around pregnancy is particularly problematic in terms of younger readers seeking practical advice via this familiar and seemingly authoritative platform, and receiving only messages about their bodies in relation to pregnancy, presented as a cis-heteronormative and passive account of the body. The example texts form a bridge between medical practitioners and everyday life, presenting an outward impression of objectivity, trustworthiness and medical authority. They lack room for nuance, present limited practical advice and reinforce a highly binary and essentialist view of womanhood, constructed as dependent on the ability to menstruate and bear children.

\section{REFERENCES}

Ahmed, S. (2017). Living a Feminist Life. Durham, North Carolina: Duke University Press. https://doi.org/10.1215/9780822373377

Ahmed, S. (2016). Interview with Judith Butler. Sexualities, 19(4), 482-492. https://doi.org/10.1177/1363460716629607

Allen, R. (2016). The Body Beyond the Anatomy Lab: (Re)Addressing Arts Methodologies for the Critical Medical Humanities, in A. Whitehead and A. Woods (eds.), The Edinburgh Companion to the Critical Medical Humanities (pp. 186-208). Edinburgh: Edinburgh University Press.

Balsamo, A. (1996). Forms of Technological Embodiment: Reading the Body in Contemporary Culture, in J. Price and M. Shildrick (eds.) (1999) Feminist Theory and the Body: A Reader (pp. 278-289). Edinburgh: Edinburgh University Press.

Bobel, C. (2006). Resistance with a Wink: Young Women, Feminism and the (Radical) Menstruating Body, in J. Lorber and L. J. Moore (eds), Gendered bodies: Feminist perspectives (pp. 87-91). Los Angeles: Roxbury Publishing Company.

Bobel, C. (2007). 'I'm not an activist, though I've done a lot of it': Doing Activism, Being Activist and the 'Perfect Standard' in a Contemporary Movement. Social Movement Studies, 6(2), 147-159. https://doi.org/10.1080/14742830701497277.

Bobel, C. (2008). From Convenience to Hazard: A Short History of the Emergence of the Menstrual Activism Movement, 1971-1992. Health Care for Women International, 29(7), 738-754. https://doi.org/10.1080/07399330802188909 
Bobel, C. (2010). New Blood: Third-Wave Feminism and the Politics of Menstruation. New Brunswick, New Jersey and London: Rutgers University Press.

Bobel, C. and Kissling E. A. (2011). Menstruation Matters: Introduction to Representations of the Menstrual Cycle. Women's Studies, 40(2), 121-126. https:/ / doi.org/10.1080/00497878.2011.537981

Boots WebMD (nd). About BootsWebMD. Boots WebMD. Available at:

https://web.archive.org/web/20180503201000/https://www.webmd.boots.com/about-webmdpolicies/default.htm?ss=ftr (Accessed 12 August 2018).

Boots WebMD (2016). Normal Menstrual Cycle and Periods. Boots WebMD Women's Health Guide, medically reviewed by Hicks, R, 5 December 2016. Available at: https://web.archive.org/web/20180328192117/https://www.webmd.boots.com/women/guide/normalmenstrual-cycle-periods (Accessed 12 August 2018).

BPAS. (2017). BPAS Comment on Boots' Failure to Meet Its Deadline for Providing Affordable Emergency Contraception. Press Release. 31 October 2017. Available at: https://www.bpas.org/about-our-charity/pressoffice/press-releases/bpas-comment-on-boots-failure-to-meet-its-deadline-for-providing-affordableemergency-contraception (Accessed 4 April 2017).

Clemmer, C. B. (2018). Rainbow Bleeding [Poem]. Bleeding While Trans Blog. 22 March 2018. Available at: https://www.bleedingwhiletrans.com/single-post/2018/03/22/Rainbow-Bleeding-Poem (Accessed 12 August 2018).

Fahs, B. (2016). Out for Blood: Essays on Menstruation and Resistance. Albany: SUNY Press.

Fassler, J. (2015). How Doctors Take Women's Pain Less Seriously. The Atlantic, 15 October 2015. Available at: https://www.theatlantic.com/health/archive/2015/10/emergency-room-wait-times-sexism/410515 (Accessed: 23 August 2017).

Fillingim, R. B., King, C. D., Ribeiro-Dasilva, M. C., Rahim-Williams, Riley III, J. L. (2009). Sex, Gender, and Pain: A Review of Recent Clinical and Experimental Findings. The Journal of Pain, 10(5), 447-485. https://doi.org/10.1016/j.jpain.2008.12.001

Fine, C. (2017). Testosterone Rex: Unmaking the Myths of our Gendered Minds. New York: Icon Books.

Hajjeh, R. A., Reingold, A. L., Weil, A., Shutt, K., Schuchat, A. and Perkins, B. A. (1999). Toxic Shock Syndrome in the United States: Surveillance Update, 1979-1996. Emerging Infectious Diseases, 5(6), 807-810. https://doi.org/10.3201/eid0506.990611

Hamberg, K. (2008). Gender Bias in Medicine. Women's Health, 4(3), 237-243. https://doi.org/10.2217/17455057.4.3.237

Haug, V. (ed.) (1987). Female Sexualization: A Collective Work of Memory. London: Verso.

Health and Social Care Information Centre. (2014). NHS Contraceptive Services: England, Community Contraceptive Clinics Statistics for 2013-14, Report, NHS Digital, Published 30 October 2014. Available at: http:// digital.nhs.uk/ catalogue/PUB15746 (Accessed 3 April 2018).

Hoffmann, D. E. and Tarzian, A. J. (2001). The Girl Who Cried Pain: A Bias against Women in the Treatment of Pain. The Journal of Law, Medicine \& Ethics, 28, 13-27. https:// doi.org/10.1111/j.1748-720X.2001.tb00037.x

Holdcroft, A. (2007). Gender bias in research: how does it affect evidence based medicine? Joumal of the Royal Society of Medicine, 100(1), 2-3. https:// doi.org/10.1177/014107680710000102

Houppert, K. (2000). The Curse: Confronting the Last Unmentionable Taboo: Menstruation. London: Profile Books Ltd.

House of Commons. (2017). Sanitary Products, Volume 632, C175WH. Available at: https://hansard.parliament.uk/Commons/2017-11-29/debates/1892E11C-640C-4B9E-A42638B1F32B4E4F/SanitaryProducts (Accessed 6 April 2018).

Hughes, B. and Petersen, E. (2017a). Un-Voiced, Soundwork. Available at: https://soundcloud.com/comfortzones (Accessed 14 April 2018).

Hughes, B. and Petersen, E. (2017b). Comfort Zones. Exhibition at Atrium Gallery, Liverpool School of Art and Design, 26 October - 3 November 2017.

Johnston-Robledo, I. and Chrisler, J. C. (2011). The Menstrual Mark: Menstruation as Social Stigma. Sex Roles, 68, 9-18. https://doi.org/10.1007/s11199-011-0052-z

Jones, A. (1998). Body Art: Performing the Subject. Minneapolis: University of Minnesota Press.

Jones, A. (ed.) (2005). The Feminism and Visual Culture Reader. Oxon and New York: Routledge.

Kristeva, J. (1982). Powers of Horror: An Essay on Abjection. New York: Columbia University Press.

Lander, L. (1988). Images of Bleeding: Menstruation as Ideology. New York: Orlando Press.

Lee, J. (1994). Menarche and the (Hetero)Sexualization of the Female Body. Gender \& Society, 8(3), 343-362. https://doi.org/10.1177/089124394008003004

MacCabe, K. and Hull, D. (2014). How Patients use Online Sources for Information. Nursing Times, 110(42), 2445.

Martin, E. (2001). The Woman in the Body: A Cultural Analysis of Reproduction. Boston: Beacon Press. 
Miles, B. (2010). William Burroughs: el Hombre Invisible. Croydon: Virgin Books.

Millard, C. and Wessely, S. (2014). Parity of Esteem between Mental and Physical Health Means Different Things to Different People, Making It Difficult To Enforce. BMJ, 349, g6821. https://doi.org/10.1136/bmj.g6821

Newton, V. L. (2016). Everyday Discourses of Menstruation: Cultural and Social Perspectives. London: Palgrave Macmillan. https://doi.org/10.1057/978-1-137-48775-9

NHS. (2015). NHS Choices - What We Do, NHS Choices, last reviewed 30 November 2015. Available at: https://www.nhs.uk/aboutNHSChoices/aboutnhschoices/Pages/what-we-do.aspx (Accessed 12 August 2018).

NHS. (2016). Periods, NHS Choices, Last Reviewed 28 July 2016. Available at: https://www.nhs.uk/conditions/periods/ (Accessed 4 April 2018)

NICE. (2014). Dysmenorrhoea, Clinical Knowledge Summaries, National Institute for Health and Care Excellence. Available at: https:/ / cks.nice.org.uk/dysmenorrhoea\#!topicsummary (Accessed 18 March 2018).

NHS Digital. (2017). Statistics on Sexual and Reproductive Health Services: England 2016/17, NHS Digital, 5 October 2017. Available at: https://digital.nhs.uk/catalogue/PUB30094 (Accessed 3 April 2018).

ONS. (2017). Childbearing for women born in different years, England and Wales: 2016, Statistical Bulletin, ONS. Available at:

https://www.ons.gov.uk/peoplepopulationandcommunity/birthsdeathsandmarriages/conceptionandfertilityr ates/bulletins/childbearingforwomenbornindifferentyearsenglandandwales/2016 (Accessed 18 March 2018)

Oudshoorn, N. (1994). Beyond the Natural Body: An Archaeology of Sex Hormones. London: Routledge.

Patient.info. (2017). Periods and Period Problems. Patient.info, authored by Tidy, C. Available at: https://.info/health/periods-and-period-problems (Accessed 18 March 2018).

Persdotter, J. (forthcoming). Introducing Menstrunormativity, in C. Bobel, B. Fahs, K. A. Hasson, E. Kissling, T. Roberts and I. Winkler (eds.), The Handbook of Critical Menstruation Studies. To be confirmed: Palgrave Macmillan.

Quint, C. (2017). I've stopped saying 'feminine hygiene products'. Here's why you should too. The Independent. Available at: https://www.independent.co.uk/voices/periods-period-poverty-tampons-menstruationempowerment-language-sanitary-a8000641.html (Accessed 14 April 2018).

Rees, E. (2017). Varieties of Embodiment and 'Corporeal Style', in E. Rees (ed.), Talking Bodies (pp. 1-15). Cham: Palgrave Macmillan. https://doi.org/10.1007/978-3-319-63778-5_1

Rose, N. (1999). Governing the Soul: The Shaping of the Private Self. London and New York: Free Association Books.

Røstvik, C. M. (2018). Cash Flow: Creativity in Menstrual Advertising, Presentation at Menstruation Symposium, University of St Andrews.

Samulowitz, A., Gremyr, I., Eriksson, E., and Hensing, G. (2018). "Brave Men” and "Emotional Women": A Theory-Guided Literature Review on Gender Bias in Health Care and Gendered Norms towards Patients with Chronic Pain. Pain Research and Management. https:/ / doi.org/10.1155/2018/6358624

Sharma, H., Smith, D., Turner, C. E., Game, L., Pichon, B., Hope, R., Kearns, A. and Sriskandan, S. (2018). Clinical and Molecular Epidemiology of Staphylococcal Toxic Shock Syndrome in the United Kingdom. Emerging Infectious Diseases, 24(2), 258-266. https:/ / doi.org/10.3201/eid2402.170606

Smart, J. M. and Burling, D. (2001). Radiology and the Internet: A Systematic Review of Patient Information Resources. Clinical Radiology, 56, 867-870. https:/ / doi.org/10.1053/crad.2001.0738

Shildrick, M. and Price, J. (eds.) (1998). Introduction, Vital Signs: Feminist Reconfigurations of the Bio/logical Body. Edinburgh: Edinburgh University Press.

Tan, S. S.-L. and Goonawardene, N. (2017). Internet Health Information Seeking and the Patient-Physician Relationship: A Systematic Review. Journal of Medical Internet Research, 19(1). https://doi.org/10.2196/jmir.5729

Telegraph Reporters (2017). Mother died after paramedics accused her of faking symptoms 'for attention', inquest hears. The Telegraph. Available at: https://www.telegraph.co.uk/news/2017/07/31/mother-died-paramedicsaccused-faking-symptoms-attention-inquest/ (Accessed 20 March 2018).

Viney, W, Callard, F. and Woods, A. (2015). Critical Medical Humanities: Embracing Entanglement, Taking Risks. Medical Humanities, 41(1), 2-7. https:/ / doi.org/10.1136/medhum-2015-010692

Wax, R. (2016). Equality 4 Mental Health. Huffington Post Blog. Available at: https:/ /www.huffingtonpost.co.uk/rubywax/equality-4-mental-health_b_8459466.html (Accessed 3 April 2013).

Young, I. (1990). Throwing Like a Girl and Other Essays in Feminist Philosophy. Bloomington: Indiana University Press.

Citation: Hughes, B. (2018). Challenging Menstrual Norms in Online Medical Advice: Deconstructing Stigma through Entangled Art Practice. Feminist Encounters: A Journal of Critical Studies in Culture and Politics, $2(2), 15$. https://doi.org/10.20897/femenc/3883

Copyright (C) 2018 by Author/s and Licensed by Lectito BV, Netherlands. This is an open access article distributed under the Creative Commons Attribution License which permits unrestricted use, distribution, and reproduction in any medium, provided the original work is properly cited. 